\title{
Triple motor mapping: transcranial, bipolar, and monopolar mapping for supratentorial glioma resection adjacent to motor pathways
}

\author{
Andrew J. Gogos, MBBS, FRACS, ${ }^{1}$ Jacob S. Young, MD, ${ }^{1}$ Ramin A. Morshed, MD, ${ }^{1}$ \\ Lauro N. Avalos, BS, ${ }^{2}$ Roger S. Noss, PhD, DABNM, ${ }^{3}$ Javier E. Villanueva-Meyer, MD, ${ }^{2}$ \\ Shawn L. Hervey-Jumper, MD, ${ }^{1}$ and Mitchel S. Berger, MD1 \\ Departments of ${ }^{1}$ Neurological Surgery and ${ }^{2}$ Radiology and Biomedical Imaging, ${ }^{3}$ Neuromonitoring Service, University of \\ California, San Francisco, California
}

\begin{abstract}
OBJECTIVE Maximal safe resection of gliomas near motor pathways is facilitated by intraoperative mapping. The authors and other groups have described the use of bipolar or monopolar direct stimulation to identify functional tissue, as well as transcranial or transcortical motor evoked potentials (MEPs) to monitor motor pathways. Here, the authors describe their initial experience using all 3 modalities to identify, monitor, and preserve cortical and subcortical motor systems during glioma surgery.
\end{abstract}

METHODS Intraoperative mapping data were extracted from a prospective registry of glioma resections near motor pathways. Additional demographic, clinical, pathological, and imaging data were extracted from the electronic medical record. All patients with new or worsened postoperative motor deficits were followed for at least 6 months.

RESULTS Between January 2018 and August 2019, 59 operations were performed in 58 patients. Overall, patients in 6 cases $(10.2 \%)$ had new or worse immediate postoperative deficits. Patients with temporary deficits all had at least Medical Research Council grade 4/5 power. Only 2 patients (3.4\%) had permanently worsened deficits after 6 months, both of which were associated with diffusion restriction consistent with ischemia within the corticospinal tract. One patient's deficit improved to $4 / 5$ and the other to $4 / 5$ proximally and $3 / 5$ distally in the lower limb, allowing ambulation following rehabilitation. Subcortical motor pathways were identified in 51 cases (86.4\%) with monopolar high-frequency stimulation, but only in 6 patients using bipolar stimulation. Transcranial or cortical MEPs were diminished in only 6 cases, 3 of which had new or worsened deficits, with 1 permanent deficit. Insula location $(p=0.001)$ and reduction in MEPs $(p=$ 0.01 ) were the only univariate predictors of new or worsened postoperative deficits. Insula location was the only predictor of permanent deficits $(p=0.046)$. The median extent of resection was $98.0 \%$.

CONCLUSIONS Asleep triple motor mapping is safe and resulted in a low rate of deficits without compromising the extent of resection.

https://thejns.org/doi/abs/10.3171/2020.3.JNS193434

KEYWORDS motor mapping; stimulation mapping; glioma; oncology

$\mathrm{T}$ HERE is compelling evidence that more extensive resection improves progression-free and overall survival for patients with glioma. ${ }^{1-11}$ However, this goal must be balanced with the need to avoid postoperative neurological deficits by preserving functional cortex and subcortical tracts. ${ }^{12-14} \mathrm{New}$ or worsened postoperative neurological deficits are associated with impaired quality of life ${ }^{15}$ and overall survival. ${ }^{16,17}$
Although preoperative imaging modalities such as blood oxygen level-dependent functional MRI, magnetoencephalography, transcranial magnetic stimulation, and MRI fiber tracking can facilitate surgical planning, direct cortical and subcortical stimulation remains the gold standard for determining function. ${ }^{18}$ Of the above techniques, only MRI tractography can reliably identify subcortical motor pathways ${ }^{19}$ however, intraoperative localization of deep

ABBREVIATIONS ADC = apparent diffusion coefficient; dcMEP = direct cortical MEP; EOR = extent of resection; HARDI = high angular resolution diffusion imaging; ISI = interstimulus interval; MEP = motor evoked potential; tcMEP = transcranial MEP.

SUBMITTED December 20, 2019. ACCEPTED March 31, 2020.

INCLUDE WHEN CITING Published online June 5, 2020; DOI: 10.3171/2020.3.JNS193434. 
pathways using neuronavigation becomes increasingly inaccurate as tumor is resected and brain shift increases. ${ }^{20}$

Bipolar stimulation is the most well-established technique for motor mapping. Initially described in humans by Wilder Penfield and colleagues in the first half of the 20th century, ${ }^{21}$ the technique has been incrementally improved over the following decades. ${ }^{13,22-24}$ More recently, a growing body of evidence supports the use of high-frequency monopolar stimulation ${ }^{25-27}$ for both cortical and subcortical mapping. While stimulation facilitates identification of functional tissue, motor evoked potentials (MEPs) can be utilized to monitor motor function during resection. This may be performed transcranially (tcMEP) ${ }^{28,29}$ or by direct cortical stimulation via a grid or strip electrode (dcMEP). ${ }^{26,28}$

While these 3 techniques have been frequently used independently or in various combinations with each other, there is a paucity of data examining postoperative motor deficits when all 3 are used together. We hypothesized that the use of bipolar stimulation, monopolar stimulation, and MEP monitoring in combination would result in a lower rate of postoperative motor deficits when compared with historical controls using one or more techniques separately. Here, we present our initial experience using triple motor mapping during intraaxial tumor resection.

\section{Methods}

\section{Patient Selection and Characteristics}

We queried our institution's prospective registry of all resections using motor mapping to identify consecutively treated patients in whom triple modality mapping (defined as bipolar and monopolar stimulation in conjunction with tcMEPs and/or dcMEPs) was utilized for glioma resection. The decision to perform awake surgery with bipolar mapping or asleep surgery with triple motor mapping was based on the site and size of the tumor, patient preference, and whether other functions were also at risk. All patients were treated at the University of California, San Francisco Medical Center between January 2018 and August 2019. Intraoperative mapping data were extracted from the registry and operative and neurophysiologists' notes. Additional demographic, clinical, pathological, and imaging data were extracted from the patients' electronic medical records. All patients with new or worsened postoperative motor deficits were followed for at least 6 months. Deficits were considered permanent if they persisted at 6 months. All motor deficits were included, regardless of severity. IRB approval was granted by the University of California, San Francisco.

\section{Anesthetic Protocol}

All motor mapping was performed under general anesthesia. One patient had awake speech mapping and was then placed under general anesthesia for motor mapping. A short-acting neuromuscular blockade was used during induction. The patient was then maintained on $50 \%-70 \%$ nitrous oxide and a volatile halogenated anesthetic (sevoflurane, desflurane, or isoflurane, less than 0.5 minimum alveolar concentration) with remifentanil. Propofol was prepared and placed in line but was only used if needed to suppress stimulation-induced seizures. Bite blocks were placed bilaterally around the endotracheal tube. The patient's temperature was maintained above $36^{\circ} \mathrm{C}$ during motor mapping. All lines were placed in the ipsilateral limb when possible, and the contralateral face, arm, and leg were exposed during motor stimulation. Mechanical venous thromboembolism prophylaxis was not applied to the contralateral leg. ${ }^{30}$

\section{Surgical and Mapping Technique}

All cases were performed using neuronavigation with overlaid corticospinal tractography. After registration, corkscrew electrodes were applied over the ipsilateral primary motor cortex based on navigation landmarks. Subdermal needle electrodes were placed in the contralateral face, upper arm, forearm, hand, upper leg, lower leg, and foot. High-frequency tcMEP monitoring was performed at intervals ranging from 1 to 5 minutes $(0-200 \mathrm{~V}, 50$ - to $500-$ usec pulse duration, $0-9$ pulses at 1 - to $4-\mathrm{msec}$ interstimulus interval [ISI]), depending on the amount of muscle movement generated by stimulation (Fig. 1A).

Bipolar cortical mapping was performed using the Ojemann stimulator (Radionics Inc.) with a 5-mm bipolar probe, delivering a biphasic square-wave pulse of 1-msec duration at $60 \mathrm{~Hz}$. During stimulation, a neurophysiologist looked for electromyography changes, and all team members directly observed the patient. Stimulation commenced at $4 \mathrm{~mA}$, and the current was incremented to a maximum of $16 \mathrm{~mA}$. Where possible, a 4-contact strip electrode was passed subdurally for dcMEP monitoring (0-20 mA, 50- to 500- $\mu$ sec pulse duration, 0-9 pulses at 1- to 4-msec ISI) after optimizing the stimulation site and parameters to elicit responses from as many electromyography channels as possible (Fig. 2B). In some cases, we were not able to perform dcMEP because of scarring from previous operations, the distance from the craniotomy to the primary motor cortex, or brain shift. We reported any reduction in MEPs by $>20 \%$.

Subcortical monopolar mapping was performed using high-frequency monopolar (0-20 mA, 500- $\mu$ sec pulse duration, 5 pulses at 4-msec ISI) and bipolar stimulation (Figs. 1B and 2C). Subcortical bipolar stimulation using the above parameters was used if deemed appropriate. In general, resection was ceased if monopolar stimulation thresholds of $5 \mathrm{~mA}$ or lower were encountered. In general, the goal of resection was to remove all the contrastenhancing tumor for enhancing lesions and the FLAIR abnormality for nonenhancing tumors. For enhancing tumors, we attempted to remove as much of the FLAIR abnormality as possible based on functional limitations.

\section{Imaging}

All patients underwent postoperative MRI within 48 hours of resection. The examination was performed on a 3.0T scanner (GE Healthcare) and included 3D T1- and T2-weighted sequences as well as a high angular resolution diffusion imaging (HARDI) sequence with the following parameters: TR $6425 \mathrm{msec}$, TE $80 \mathrm{msec}, 50$ axial slices, 2-mm slice thickness, b value $=2000 \mathrm{sec} / \mathrm{mm}^{2}$, and 55 diffusion gradients. 


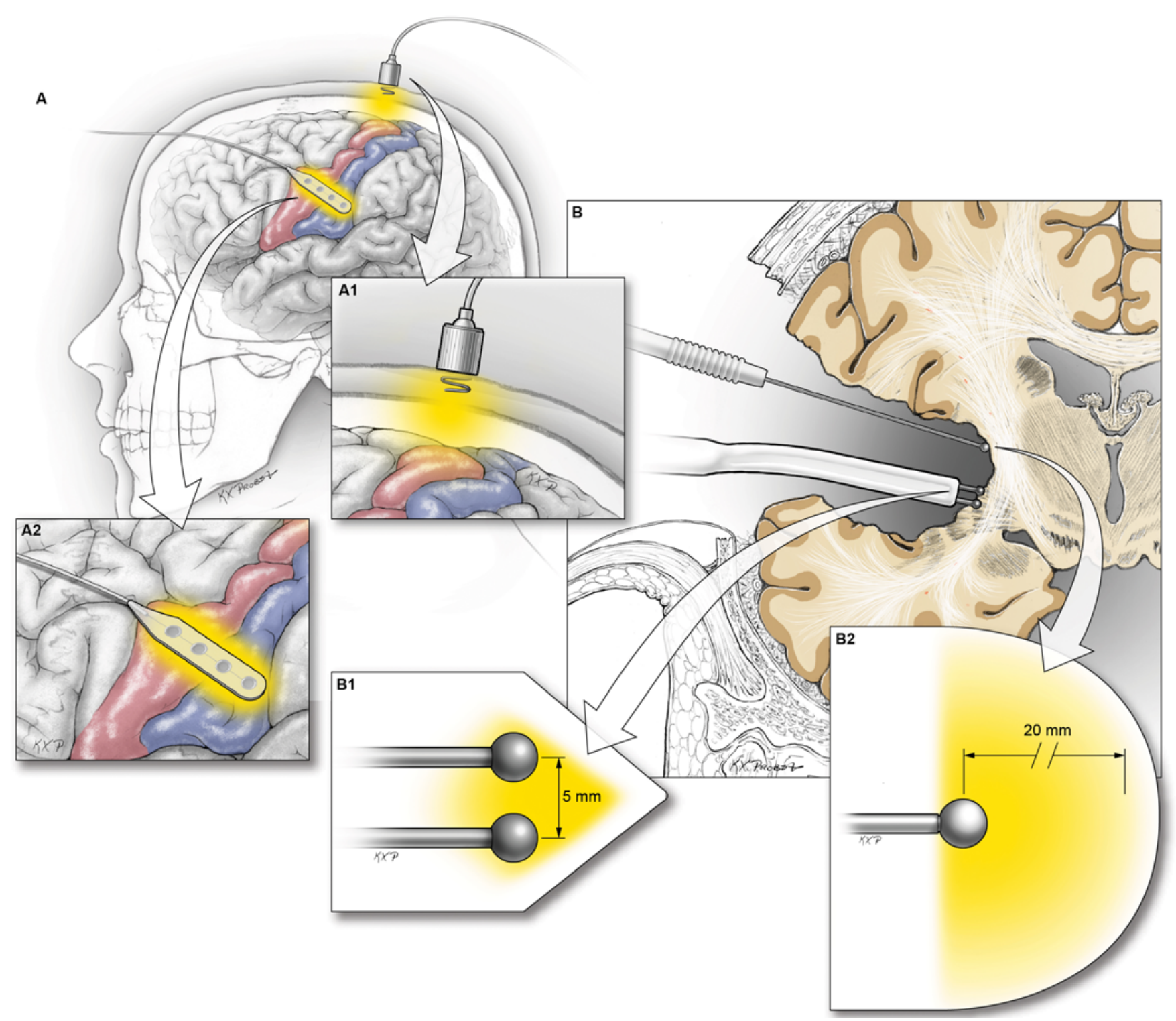

FIG. 1. Illustrations of the triple motor mapping technique. A: Illustration of monitoring with tcMEP and dcMEP electrodes. B: Illustration of subcortical mapping and current spread from bipolar and monopolar probes. Copyright Kenneth X. Probst. Published with permission.

Preoperative and postoperative tumor volumes were calculated using the Brainlab Elements SmartBrush tool (version 2.6). For contrast-enhancing tumors, the enhancing component on the T1-weighted sequence was measured, whereas for nonenhancing tumors, volumes were measured using the FLAIR signaling abnormality.

For patients with postoperative deficits, the postoperative MRI scan was reviewed to identify any areas of diffusion-weighted imaging and corresponding apparent diffusion coefficient (ADC) abnormality to suggest ischemic injury.

Corticospinal tractography was performed using methods we have previously published. ${ }^{31}$ In brief, HARDI data and anatomical sequences were analyzed offline using the AW Server (General Electric). A seed region of interest and a target region of interest were placed on the cerebral peduncle and primary motor cortex ipsilateral to the surgical cavity; fiber tracking employed a fractional anisotropy threshold of 0.15 and a tract angular change of $60^{\circ}$. Tracked white matter fibers were included as an object on the 3D T1-weighted MR images, and the shortest distance between the tract and surgical cavity was determined by a neuroradiologist (J.E.V.M.).

\section{Statistical Analysis}

The statistical significance of univariate predictors of postoperative deficits was determined using the Fisher exact test. The Student t-test and the Mann-Whitney U-test were used for parametric and nonparametric continuous variables, respectively. Pearson's correlation coefficient was used to determine the strength of the relationship be- 

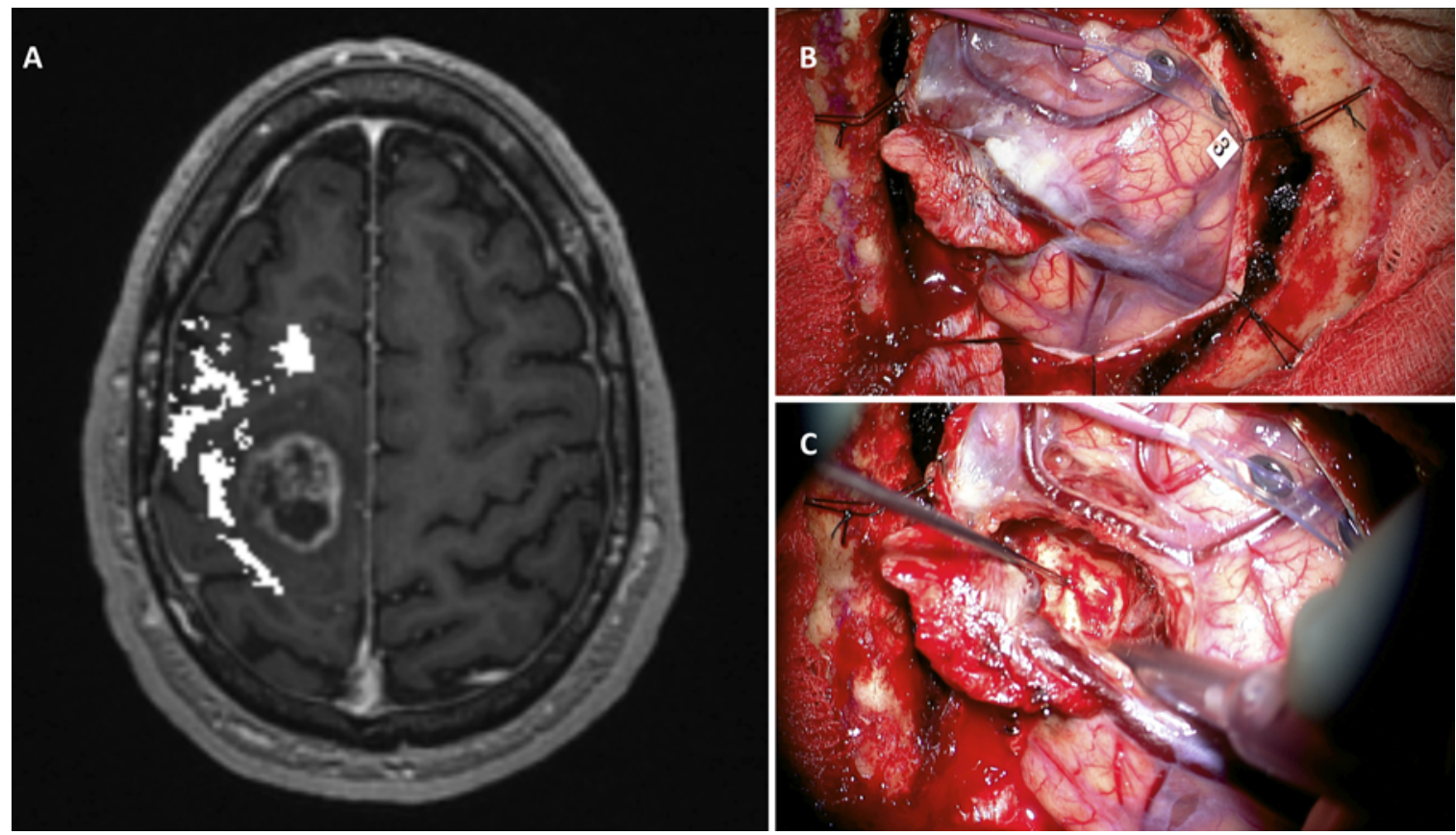

FIG. 2. Example case of triple motor mapping. A: Axial contrast-enhanced T1-weighted MR image demonstrating a precentral tumor in a 60-year-old patient with progressive left leg weakness and focal seizures, with overlaid corticospinal tractography. B: Intraoperative photograph before resection, demonstrating a positive bipolar stimulation site (3) and a 4-contact electrode for dcMEP. C: Intraoperative photograph demonstrating subcortical monopolar mapping during tumor resection.

tween monopolar stimulation and the distance to the corticospinal tract. All statistical analyses were performed in $\mathrm{R}$ (version 3.5.3, r-project.org).

\section{Results}

\section{Patient Characteristics and Mapping Details}

Between January 2018 and August 2019, 59 operations utilizing triple motor mapping were performed in 58 patients. One patient underwent a second operation for recurrence 14 months after their initial operation. Patient demographics are presented in Table 1 . Only 2 patients (3.4\%) had intraoperative seizures, which were terminated rapidly with cold irrigation. In both cases, mapping could resume after termination of the event.

Although tcMEP monitoring was performed in all cases, dcMEP monitoring was possible in only $45.8 \%$ of cases. The use of dcMEP was limited by scarring from previous operations, by the distance from the craniotomy to the primary motor cortex, or brain shift. MEPs were noted to irreversibly diminish in 6 cases $(10.2 \%)$. One patient had loss of foot responses that were associated with transiently worsened mild weakness (patient 4 , Table 2). In the other cases, the stimulation amplitude had to be increased $20 \%-40 \%$ for the same effect. One of these patients had mild transient weakness (patient 2), and another had moderate permanent weakness related to an infarct (patient 6). The other 3 changes in MEPs were likely artifactual, related to brain shift away from the inner table. The negative predictive value for MEP monitoring was $94.3 \%$, but the positive predictive value was only $50 \%$.

Subcortical mapping was performed using a combina- tion of bipolar and monopolar stimulation. Descending motor pathways were identified using monopolar stimulation in 51 cases $(86.4 \%)$. No deficits occurred in patients in whom a monopolar threshold could not be identified. Subcortical bipolar stimulation was utilized in 20 cases but was only positive in $6(30 \%)$, all of whom had monopolar stimulation thresholds of less than $10 \mathrm{~mA}$. Using a distance threshold of $5 \mathrm{~mm}$, the positive and negative predictive values of monopolar stimulation were $49.0 \%$ and $88.0 \%$, respectively.

Postoperative HARDI tractography was available in 50 cases $(84.7 \%)$, and the motor pathways could be stimulated in $43(86.0 \%)$ of these. All resections stopped at least 2 $\mathrm{mm}$ away from the corticospinal tract based on postoperative tractography. In the 7 cases in which motor pathways could not be stimulated (up to $20 \mathrm{~mA}$ ), the mean distance to the corticospinal pathway was $19.0 \mathrm{~mm}$ (range 15-22 $\mathrm{mm}$ ). There was a strong linear relationship between the amplitude required for monopolar stimulation and the distance from the resection cavity to the motor pathways (Pearson's correlation coefficient 0.77, $\mathrm{p}<0.0001$; Fig. 3). Using the derived regression formula, stimulation at 5,10 , 15 , and $20 \mathrm{~mA}$ equated with distances of 5.7, 9.5, 13.3 and $23.8 \mathrm{~mm}$, respectively.

\section{Postoperative Neurological Deficits and EOR}

Patients in 6 cases $(10.2 \%)$ had new or worse immediate postoperative motor deficits. However, only 2 patients (3.4\%) had deficits that did not recover back to baseline by 6 months (Table 2). All 4 transient deficits were mild at discharge. The persistent deficits in the 2 patients were initially severe; however, they improved substantially. At 
TABLE 1. Patient demographics and tumor characteristics

\begin{tabular}{|c|c|}
\hline Characteristic & Value \\
\hline Total no. of patients & 58 \\
\hline No. of operations & 59 \\
\hline \multicolumn{2}{|l|}{ Sex } \\
\hline Female & $28(48.3)$ \\
\hline Male & $30(51.7)$ \\
\hline Median (range) age at op, yrs & $50(17-81)$ \\
\hline \multicolumn{2}{|l|}{ Side of tumor } \\
\hline $\mathrm{Lt}$ & $15(25.4)$ \\
\hline $\mathrm{Rt}$ & $44(74.6)$ \\
\hline \multicolumn{2}{|l|}{ Site of tumor } \\
\hline Frontal & $18(30.5)$ \\
\hline Temporal & $11(18.6)$ \\
\hline Parietal & $12(20.3)$ \\
\hline Insula & 7 (11.9) \\
\hline Multilobe* & $11(18.6)$ \\
\hline \multicolumn{2}{|l|}{ Preop motor deficit } \\
\hline Yes & $22(37.3)$ \\
\hline No & $37(62.7)$ \\
\hline \multicolumn{2}{|l|}{ WHO grade } \\
\hline I & $1(1.7)$ \\
\hline II & $12(20.3)$ \\
\hline III & $9(15.3)$ \\
\hline IV & $37(62.7)$ \\
\hline \multicolumn{2}{|l|}{ Histology } \\
\hline Glioblastoma & $37(62.7)$ \\
\hline Anaplastic oligodendroglioma & $4(6.8)$ \\
\hline Anaplastic astrocytoma & $4(6.8)$ \\
\hline Oligodendroglioma & $7(11.9)$ \\
\hline Diffuse astrocytoma & $4(6.8)$ \\
\hline Other† & $3(5.1)$ \\
\hline
\end{tabular}

Values represent the number (\%) unless stated otherwise.

${ }^{*}$ Six cases included the insula.

† Ependymoma, anaplastic pleomorphic xanthoastrocytoma, and anaplastic pilocytic astrocytoma.

last follow-up, patient 5 had a facial droop, with 4/5 upperlimb weakness and normal lower-limb power. Patient 6 had 4+/5 upper-limb and 4/5 lower-limb weakness proximally and 3/5 distally and was ambulant with an orthosis and able to perform on tandem gait testing. Both patients with permanent deficits had new areas of diffusion restriction in or adjacent to the corticospinal tracts on postoperative imaging (Fig. 4).

Insula location $(p=0.001)$ and reduction in MEPs $(p=$ 0.01 ) during resection were the only univariate predictors of a new or worse postoperative deficit (Table 3 ). Insula location $(\mathrm{p}=0.046)$ was the only predictor of permanent deficits.

Postoperative volumetric imaging was available for all patients. The median extent of resection (EOR) was $98.2 \%$. Overall, $100 \%$ resection, $>90 \%$ resection, and >

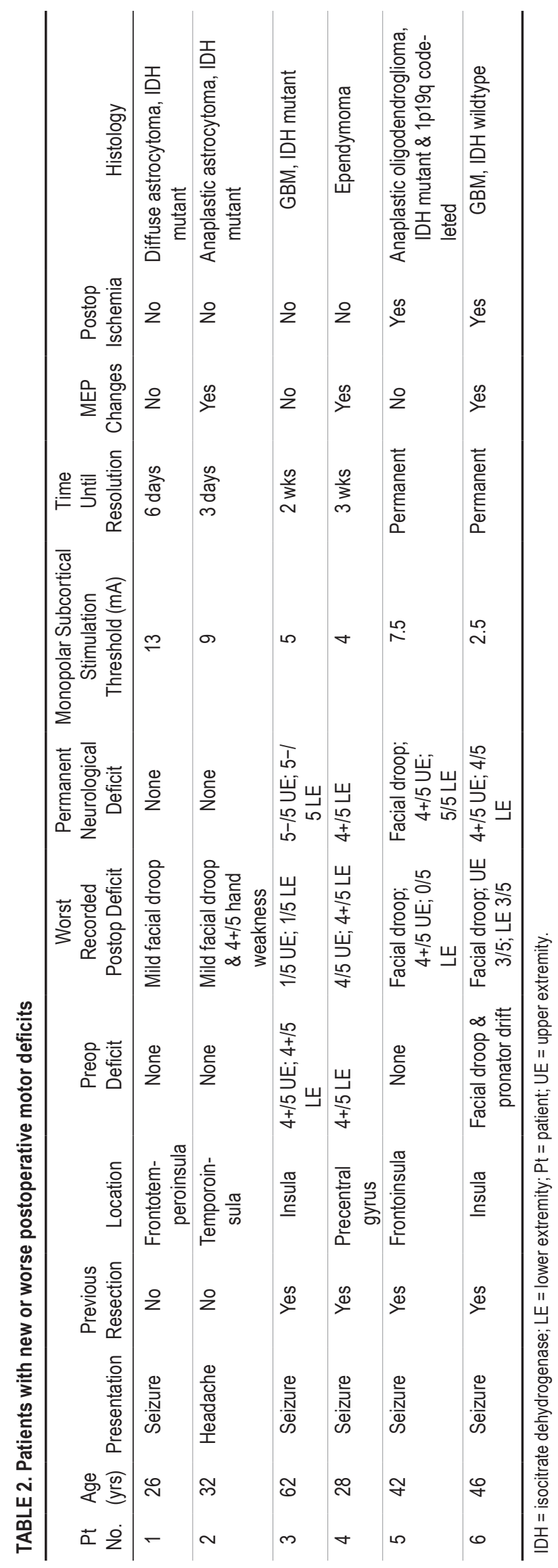




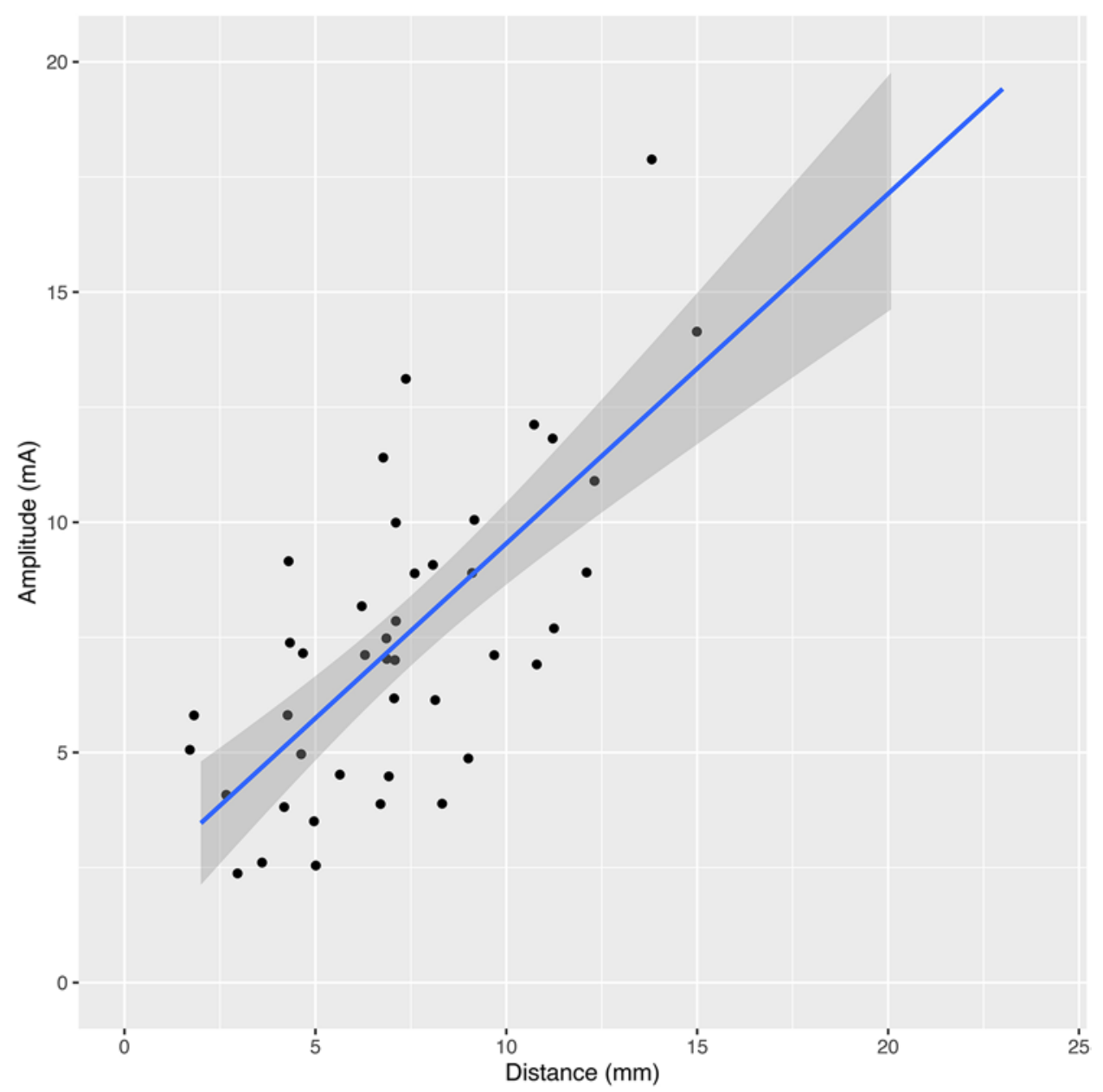

FIG. 3. Monopolar stimulation thresholds and distance to the corticospinal tracts. Scatterplot and regression line (with $95 \%$ confidence intervals) demonstrating the relationship between stimulation intensity during monopolar mapping and the distance to the motor fibers, as measured on postoperative tractography. Pearson's correlation coefficient $0.77(p<0.0001)$. Figure is available in color online only.

$70 \%$ resection were achieved in $25(42.4 \%), 45(76.3 \%)$, and $54(91.5 \%)$ cases, respectively. There was no difference in EOR between patients with postoperative deficits and those without $(\mathrm{p}=0.65)$. Likewise, there was no association between positive mapping and EOR $(p=0.66)$.

\section{Discussion}

\section{Major Findings}

Our results demonstrate the safety of resecting tumors near motor pathways using triple motor mapping. Overall, patients in 6 cases $(10.2 \%)$ had new or worsened postoperative motor function. Only 2 patients (3.4\%) had permanent postoperative deficits, both of which were attributable to stroke rather than direct motor injury. There were no cases of severe permanent deficits. The risk of a deficit was higher in patients with insular involvement and in those in whom there was a change in tcMEP or dcMEP recordings. Subcortical motor pathways were identified in $86.4 \%$ of patients with monopolar stimulation, but in only $30.0 \%$ of cases in which subcortical bipolar stimulation was attempted.

\section{Adding Subcortical High-Frequency Monopolar Stimulation}

This paper was derived from a dissatisfaction with current methods of motor mapping. In the senior author's recent series of 702 motor mapping cases using bipolar stimulation, ${ }^{12}$ descending pathways could only be identified in $43 \%$ of cases; $30 \%$ of patients had new or worsened motor deficits, and $7 \%$ had persistent deficits, one-third of which were severe (2/5 strength or worse). Only $41 \%$ of patients with permanent deficits had significant areas of restricted diffusion on postoperative MRI, suggesting that their deficits were due to direct transgression of the motor system. Our current results demonstrate a much greater ability to identify the motor system and to preserve motor function in the asleep setting, although we did not perform a direct statistical comparison.

Current spread from monopolar and bipolar electrodes is distinct. Bipolar charge is transmitted from one arm of the probe to the other. The electrical field rapidly dissipates beyond the probe but activates tissue between the arms (Fig. 1B). ${ }^{32}$ The technique is ideal for cortical stim- 

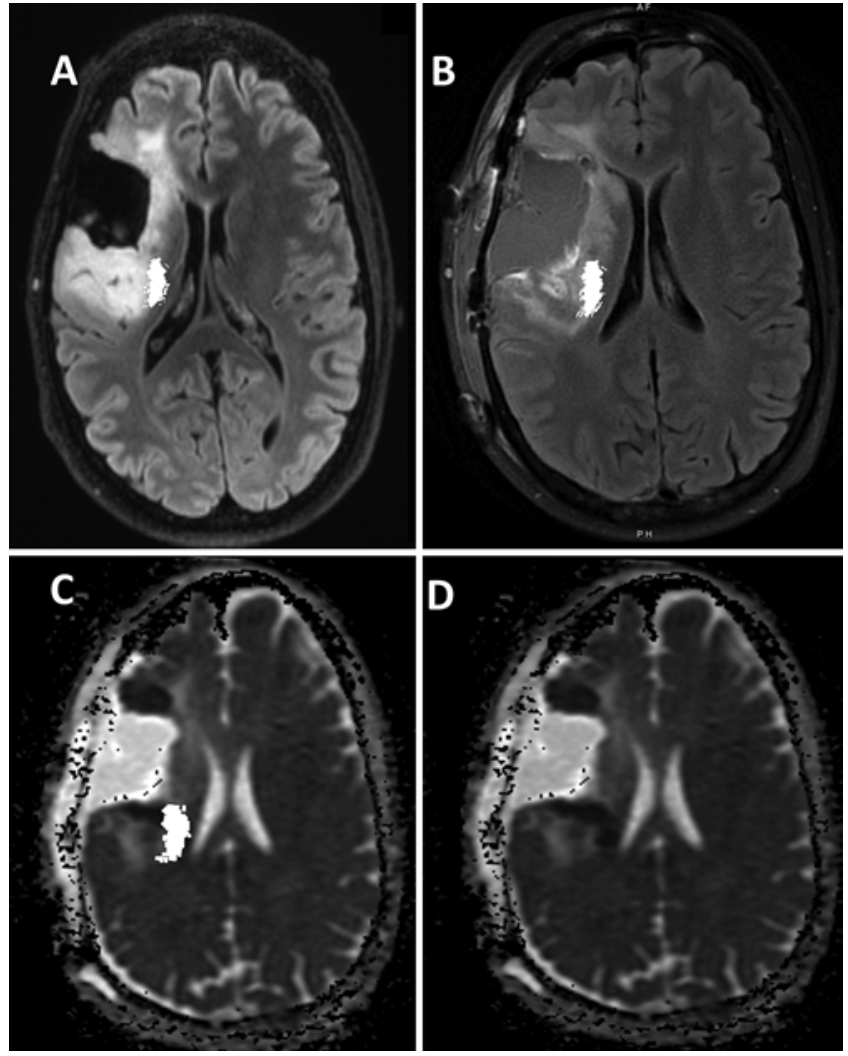

FIG. 4. Patient 5. Postoperative diffusion restriction in a 42-year-old patient who underwent re-resection of a frontoinsular anaplastic oligodendroglioma and had a permanent deficit. A: Preoperative axial FLAIR MR image with overlaid tractography. The primary aim of surgery was to resect a new enhancing nodule, and the FLAIR abnormality was debulked until the subcortical monopolar threshold was $5 \mathrm{~mA}$. B: Postoperative axial FLAIR sequence with overlaid tractography, demonstrating resection of the posterior insular nonenhancing tumor and a 4-mm distance from the corticospinal tract to the cavity. $\mathbf{C}$ and $\mathbf{D}$ : Postoperative ADC maps, with (C) and without (D) overlaid tractography, demonstrating ischemia within the corticospinal tract.

ulation where the goal is to answer the binary question: is the underlying cortex functional or not? We utilized bipolar polar cortical stimulation to identify motor cortex for dcMEP monitoring and also to identify function-free corridors to access the tumor.

In contrast to a bipolar probe, charge from a monopolar electrode spreads radially from the tip, toward a distant reference electrode (Fig. 1B). ${ }^{32}$ This spread allows the surgeon to modulate the stimulator current to estimate the distance to motor pathways. This both increases the sensitivity of mapping and allows greater spatial resolution.

Szelényi et al. ${ }^{33}$ directly compared bipolar and monopolar subcortical stimulation in 20 patients. Stimulation was positive at $92 \%$ of sites using monopolar but only at $54 \%$ of sites using a bipolar probe. Similarly, we were able to elicit monopolar stimulation in $86.4 \%$ of cases; however, only in $30.0 \%$ of cases using the bipolar probe and in all cases in which bipolar stimulation was possible was the monopolar threshold less than $10 \mathrm{~mA}$.

Several groups have previously demonstrated that the current required for monopolar subcortical stimulation in milliamps roughly approximates the distance to motor pathways in millimeters. ${ }^{34-38}$ Thus, it provides an earlier warning of proximity to motor pathways and also quantifies the distance. We have demonstrated a high correlation between distance and current using monopolar stimulation, with the 2 numbers being approximately equal.

Subcortical bipolar stimulation does not conform to the same relationship. Zhu et al. demonstrated that the distance of positive bipolar stimulation sites from motor pathways (based on intraoperative tractography) ranged from 2.0 to $14.7 \mathrm{~mm}$ with a mean of $5.2 \mathrm{~mm} .{ }^{39}$ Similarly, we found that in most cases subcortical bipolar stimulation was not possible, and, when it was, the distance to motor fibers varied from 2 to $11 \mathrm{~mm}$ with a mean of $6.3 \mathrm{~mm}$. Additionally, lack of bipolar stimulation may be falsely reassuring. There were 2 cases in our series with negative bipolar stimulation, despite the stimulation point being within $5 \mathrm{~mm}$ of the motor pathways.

\section{Adding tcMEP and dcMEP Monitoring}

Although commonly employed for brainstem and spinal cord procedures, there is more limited evidence for the role of MEP monitoring for supratentorial brain tumor resection.

Taniguchi et al. ${ }^{25}$ utilized direct high-frequency (300$500 \mathrm{~Hz}$ ) stimulation of the motor cortex, demonstrating that responses could be achieved at much lower voltages than with lower-frequency $(50-60 \mathrm{~Hz})$ stimulation. Kombos et al. ${ }^{40}$ performed dcMEP in 70 patients and demonstrated that irreversible changes in latency or loss of potentials was associated with postoperative neurological deficits. Reversible changes in MEPs were often due to retraction or movement of the electrode. Most cases of MEP deterioration are reversible, and permanent loss is associated with a high risk of permanent deficit from stroke. ${ }^{28}$ This raises the concern that, rather than providing a warning, signal alterations may occur after irreversible damage to motor pathways has already occurred. ${ }^{41}$ The high negative predictive value (94.3\%) and low positive predictive value (50\%) of MEP monitoring emphasize that it should be seen as a screening tool for damage to motor pathways rather than as a diagnostic tool. Thus, the value of MEP monitoring may be that it reassures the surgeons that the motor system is intact and that resection may proceed. However, false negatives (i.e., new deficits occurring despite stable monitoring) may occur in up to $14 \%$ of cases..$^{29,40}$ This has been attributed to postoperative edema or hemorrhage. In our study the false-negative rate was $5.7 \%$, with 1 permanent deficit attributable to stroke (which may have occurred after cessation of monitoring) and 2 mild transient deficits without a clear cause. In all 3 cases, monitoring was performed with tcMEP rather than dcMEP.

We utilized tcMEP monitoring more than other groups, ${ }^{28,42}$ possibly because of scarring from previous resections or our preference for small craniotomies and negative mapping, making direct cortical stimulation more difficult.

\section{New or Worse Deficits and EOR}

Immediate postoperative deficits may occur in up to $60 \% 12,28,43$ of resections of tumors in or near motor pathways. Indeed, transient deficits may be associated with 
TABLE 3. Univariate predictors of new or worse motor deficits (transient or permanent)

\begin{tabular}{|c|c|c|c|}
\hline Variable & Total & Deficit & p Value \\
\hline Number of operations & 59 & $6(10.2)$ & \\
\hline Mean age, yrs & 50.3 & 39.6 & 0.06 \\
\hline Sex & & & 0.67 \\
\hline Female & $28(48.3)$ & $2(7.1)$ & \\
\hline Male & $30(51.7)$ & $4(13.3)$ & \\
\hline Side & & & $>0.99$ \\
\hline $\mathrm{Lt}$ & $15(25.4)$ & $1(6.7)$ & \\
\hline $\mathrm{Rt}$ & $44(74.6)$ & $5(11.4)$ & \\
\hline Site & & & 0.001 \\
\hline Insula & $13(22.0)$ & $5(38.5)$ & \\
\hline Not involving insula & $46(80.0)$ & $1(2.2)$ & \\
\hline Preop motor deficit & & & 0.66 \\
\hline Yes & $22(37.3)$ & $3(13.6)$ & \\
\hline No & $37(62.7)$ & $3(8.1)$ & \\
\hline Previous resection & & & $>0.99$ \\
\hline Yes & $34(57.6)$ & $4(11.8)$ & \\
\hline No & $25(42.4)$ & $2(8.0)$ & \\
\hline High-grade glioma & & & 0.60 \\
\hline Yes & $46(78.0)$ & $4(8.7)$ & \\
\hline No & $13(22.0)$ & $2(15.4)$ & \\
\hline Decrease or loss in MEP & & & 0.01 \\
\hline Yes & $6(10.2)$ & $3(50.0)$ & \\
\hline No & $53(89.8)$ & $3(5.7)$ & \\
\hline Monopolar threshold $\leq 5 \mathrm{~mA}$ & & & 0.34 \\
\hline Yes & $18(30.5)$ & $3(16.7)$ & \\
\hline No & $41(69.5)$ & $3(7.3)$ & \\
\hline
\end{tabular}

Boldface type indicates statistical significance.

improved survival, ${ }^{16}$ likely because of a greater EOR. Permanent deficits, however, may be associated with worse survival ${ }^{16,17}$ and quality of life. ${ }^{15}$ Improvements in monitoring and mapping techniques have decreased the rate of permanent deficits. The rate of permanent deficits in studies utilizing a single monitoring or mapping modality has been reported to be between $7 \%$ and $12.5 \% .^{12,28,39}$ Groups utilizing both MEP monitoring and monopolar subcortical stimulation $^{26,41,42}$ have demonstrated rates of permanent neurological deficits of $5 \%$ or less. In the present series utilizing triple modality mapping, permanent deficits occurred in 2 patients $(3.4 \%)$, both of which were due to ischemia rather than direct transgression of the motor system. Other than insula location, there were no statistically significant predictors of permanent deficits, likely because of the rarity of this event.

We have previously reported median EORs of $96 \%$ and $88 \%$ for first resection of glioblastoma ${ }^{5}$ and low-grade glioma, ${ }^{6}$ respectively. In this series, we achieved a comparable median EOR of $98.2 \%$, despite the proximity to motor pathways and the high proportion of recurrent tumors.

\section{Limitations and Generalizability}

Although cases were identified from a prospective reg- istry, this is a retrospective study and limited by the quality of data collected within the medical record. Documentation of motor outcome was based on inpatient notes, discharge summaries, and clinic letters written by the treating residents, neurosurgeons, and neurooncologists. It is possible that examination by an independent examiner may have revealed subtle deficits that were missed during these visits. Although we have demonstrated significantly improved clinical outcomes when compared with our previous mapping technique, it is possible that these results are in part due to other factors, such as greater experience or increased use of navigated tractography.

These are the results of subspecialist tumor neurosurgeons, with a dedicated anesthesia and neuromonitoring team working in a high-volume center, and they may thus not be generalizable to all surgeons and institutions. However, the principles utilized may be learned by any experienced neurosurgeon and integrated into practice.

\section{Conclusions}

Transcranial and direct cortical MEP monitoring combined with bipolar and monopolar stimulation resulted in improved localization of functional tissue and low rates of transient and permanent deficits. Monopolar stimula- 
tion could reliably predict the distance to the corticospinal tract. All permanent postoperative deficits were related to ischemia rather than direct injury to motor pathways. Compared with our previous experience with subcortical bipolar stimulation to localize deep motor tracts, the triad of triple motor mapping is superior in terms of enhancing efficient resection while minimizing motor deficits.

\section{Acknowledgments}

We thank Kenneth X. Probst for illustrating the triple mapping technique in Figure 1.

Partial funding for this study was provided by the Robert Wood Johnson Foundation (grant no. 74259) to S.H.J. and NINDS (grant no. K08 110919-01) to S.H.J.

\section{References}

1. Keles GE, Anderson B, Berger MS. The effect of extent of resection on time to tumor progression and survival in patients with glioblastoma multiforme of the cerebral hemisphere. Surg Neurol. 1999;52(4):371-379.

2. Keles GE, Chang EF, Lamborn KR, et al. Volumetric extent of resection and residual contrast enhancement on initial surgery as predictors of outcome in adult patients with hemispheric anaplastic astrocytoma. J Neurosurg. 2006;105(1):34-40.

3. Lacroix M, Abi-Said D, Fourney DR, et al. A multivariate analysis of 416 patients with glioblastoma multiforme: prognosis, extent of resection, and survival. J Neurosurg. 2001;95(2):190-198.

4. McGirt MJ, Chaichana KL, Gathinji M, et al. Independent association of extent of resection with survival in patients with malignant brain astrocy toma. J Neurosurg. 2009;110(1):156-162.

5. Sanai N, Polley MY, McDermott MW, et al. An extent of resection threshold for newly diagnosed glioblastomas. $J$ Neurosurg. 2011;115(1):3-8.

6. Smith JS, Chang EF, Lamborn KR, et al. Role of extent of resection in the long-term outcome of low-grade hemispheric gliomas. J Clin Oncol. 2008;26(8):1338-1345.

7. Stummer W, Reulen HJ, Meinel T, et al. Extent of resection and survival in glioblastoma multiforme: identification of and adjustment for bias. Neurosurgery. 2008;62(3):564-576.

8. Ushio Y, Kochi M, Hamada J, et al. Effect of surgical removal on survival and quality of life in patients with supratentorial glioblastoma. Neurol Med Chir (Tokyo). 2005;45(9):454461.

9. Vecht CJ, Avezaat CJJ, van Putten WLJ, et al. The influence of the extent of surgery on the neurological function and survival in malignant glioma. A retrospective analysis in 243 patients. J Neurol Neurosurg Psychiatry. 1990;53(6):466-471.

10. Lamborn KR, Chang SM, Prados MD. Prognostic factors for survival of patients with glioblastoma: recursive partitioning analysis. Neuro Oncol. 2004;6(3):227-235.

11. Jeremic B, Grujicic D, Antunovic V, et al. Influence of extent of surgery and tumor location on treatment outcome of patients with glioblastoma multiforme treated with combined modality approach. J Neurooncol. 1994;21(2):177-185.

12. Han SJ, Morshed RA, Troncon I, et al. Subcortical stimulation mapping of descending motor pathways for perirolandic gliomas: assessment of morbidity and functional outcome in 702 cases. J Neurosurg. 2018;131(1):201-208.

13. Duffau H, Capelle L, Denvil D, et al. Usefulness of intraoperative electrical subcortical mapping during surgery for low-grade gliomas located within eloquent brain regions: functional results in a consecutive series of 103 patients. $J$ Neurosurg. 2003;98(4):764-778.
14. Keles GE, Lundin DA, Lamborn KR, et al. Intraoperative subcortical stimulation mapping for hemispherical perirolandic gliomas located within or adjacent to the descending motor pathways: evaluation of morbidity and assessment of functional outcome in 294 patients. J Neurosurg. 2004;100(3):369-375.

15. Chaichana KL, Halthore AN, Parker SL, et al. Factors involved in maintaining prolonged functional independence following supratentorial glioblastoma resection. Clinical article. J Neurosurg. 2011;114(3):604-612.

16. Rahman M, Abbatematteo J, De Leo EK, et al. The effects of new or worsened postoperative neurological deficits on survival of patients with glioblastoma. J Neurosurg. 2017;127(1):123-131.

17. McGirt MJ, Mukherjee D, Chaichana KL, et al. Association of surgically acquired motor and language deficits on overall survival after resection of glioblastoma multiforme. Neurosurgery. 2009;65(3):463-470.

18. Tarapore PE, Tate MC, Findlay AM, et al. Preoperative multimodal motor mapping: a comparison of magnetoencephalography imaging, navigated transcranial magnetic stimulation, and direct cortical stimulation. J Neurosurg. 2012;117(2):354-362.

19. Wu JS, Zhou LF, Tang WJ, et al. Clinical evaluation and follow-up outcome of diffusion tensor imaging-based functional neuronavigation: a prospective, controlled study in patients with gliomas involving pyramidal tracts. Neurosurgery. 2007;61(5):935-949.

20. Nimsky C, Ganslandt O, Hastreiter P, et al. Intraoperative diffusion-tensor MR imaging: shifting of white matter tracts during neurosurgical procedures-initial experience. Radiology. 2005;234(1):218-225.

21. Penfield W, Boldrey E. Somatic motor and sensory representation in man. Brain. 1937;60(4):389-443.

22. Bello L, Gambini A, Castellano A, et al. Motor and language DTI Fiber Tracking combined with intraoperative subcortical mapping for surgical removal of gliomas. Neuroimage. 2008;39(1):369-382.

23. Berger MS. Functional mapping-guided resection of lowgrade gliomas. Clin Neurosurg. 1995;42:437-452.

24. Berger MS, Ojemann GA. Intraoperative brain mapping techniques in neuro-oncology. Stereotact Funct Neurosurg. 1992;58(1-4):153-161.

25. Taniguchi M, Cedzich C, Schramm J. Modification of cortical stimulation for motor evoked potentials under general anesthesia: technical description. Neurosurgery. 1993;32(2):219-226.

26. Schucht P, Seidel K, Beck J, et al. Intraoperative monopolar mapping during 5-ALA-guided resections of glioblastomas adjacent to motor eloquent areas: evaluation of resection rates and neurological outcome. Neurosurg Focus. 2014;37(6):E16.

27. Raabe A, Beck J, Schucht P, Seidel K. Continuous dynamic mapping of the corticospinal tract during surgery of motor eloquent brain tumors: evaluation of a new method. J Neurosurg. 2014;120(5):1015-1024.

28. Neuloh G, Pechstein U, Schramm J. Motor tract monitoring during insular glioma surgery. J Neurosurg. 2007;106(4):582592.

29. Lee JJ, Kim YI, Hong JT, et al. Intraoperative monitoring of motor-evoked potentials for supratentorial tumor surgery. $J$ Korean Neurosurg Soc. 2014;56(2):98-102.

30. Auguste KI, Quinones-Hinojosa A, Gadkary C, et al. Incidence of venous thromboembolism in patients undergoing craniotomy and motor mapping for glioma without intraoperative mechanical prophylaxis to the contralateral leg. $J$ Neurosurg. 2003;99(4):680-684.

31. Caverzasi E, Hervey-Jumper SL, Jordan KM, et al. Identifying preoperative language tracts and predicting postoperative functional recovery using HARDI q-ball fiber tractography in patients with gliomas. J Neurosurg. 2016;125(1):33-45. 
32. Pallud J, Mandonnet E, Corns R, et al. Technical principles of direct bipolar electrostimulation for cortical and subcortical mapping in awake craniotomy. Neurochirurgie. 2017;63(3):158-163.

33. Szelényi A, Senft C, Jardan M, et al. Intra-operative subcortical electrical stimulation: a comparison of two methods. Clin Neurophysiol. 2011;122(7):1470-1475.

34. Maesawa S, Fujii M, Nakahara N, et al. Intraoperative tractography and motor evoked potential (MEP) monitoring in surgery for gliomas around the corticospinal tract. World Neurosurg. 2010;74(1):153-161.

35. Kamada K, Todo T, Ota T, et al. The motor-evoked potential threshold evaluated by tractography and electrical stimulation. J Neurosurg. 2009;111(4):785-795.

36. Ohue S, Kohno S, Inoue A, et al. Accuracy of diffusion tensor magnetic resonance imaging-based tractography for surgery of gliomas near the pyramidal tract: a significant correlation between subcortical electrical stimulation and postoperative tractography. Neurosurgery. 2012;70(2):283-294.

37. Prabhu SS, Gasco J, Tummala S, et al. Intraoperative magnetic resonance imaging-guided tractography with integrated monopolar subcortical functional mapping for resection of brain tumors. Clinical article. J Neurosurg. 2011;114(3):719726.

38. Shiban E, Krieg SM, Haller B, et al. Intraoperative subcortical motor evoked potential stimulation: how close is the corticospinal tract? J Neurosurg. 2015;123(3):711-720.

39. Zhu FP, Wu JS, Song YY, et al. Clinical application of motor pathway mapping using diffusion tensor imaging tractography and intraoperative direct subcortical stimulation in cerebral glioma surgery: a prospective cohort study. Neurosurgery. 2012;71(6):1170-1184.

40. Kombos T, Suess O, Ciklatekerlio O, Brock M. Monitoring of intraoperative motor evoked potentials to increase the safety of surgery in and around the motor cortex. J Neurosurg. 2001;95(4):608-614.
41. Seidel K, Beck J, Stieglitz L, et al. The warning-sign hierarchy between quantitative subcortical motor mapping and continuous motor evoked potential monitoring during resection of supratentorial brain tumors. J Neurosurg. 2013;118(2):287296.

42. Moiyadi A, Velayutham P, Shetty P, et al. Combined motor evoked potential monitoring and subcortical dynamic mapping in motor eloquent tumors allows safer and extended resections. World Neurosurg. 2018;120:e259-e268.

43. Magill ST, Han SJ, Li J, Berger MS. Resection of primary motor cortex tumors: feasibility and surgical outcomes. $J$ Neurosurg. 2018;129(4):961-972.

\section{Disclosures}

The authors report no conflict of interest concerning the materials or methods used in this study or the findings specified in this paper.

\section{Author Contributions}

Conception and design: Gogos, Hervey-Jumper, Berger. Acquisition of data: Gogos, Young, Morshed, Avalos, Noss, Villanueva-Meyer, Berger. Analysis and interpretation of data: Gogos, Young, Morshed, Noss, Villanueva-Meyer, Berger. Drafting the article: Gogos. Critically revising the article: Gogos, Young, Morshed, Noss, Villanueva-Meyer, Hervey-Jumper, Berger. Reviewed submitted version of manuscript: Gogos, Young, Morshed, Avalos, Villanueva-Meyer, Hervey-Jumper, Berger. Approved the final version of the manuscript on behalf of all authors: Gogos. Statistical analysis: Gogos. Study supervision: Hervey-Jumper, Berger.

\section{Correspondence}

Andrew J. Gogos: University of California, San Francisco, CA. andrew.gogos@ucsf.edu. 